\title{
The ATLAS TileCal Read-Out Drivers Signal Reconstruction
}

\author{
Alberto Valero \\ IFIC (CSIC E Universidad de Valencia) \\ On Behalf Of The ATLAS Tile Calorimeter Group
}

\section{Introduction}

TileCal is the hadronic tile calorimeter of the ATLAS experiment at LHC/CERN. The main component of the TileCal back-end electronics is the Read-Out Driver (ROD). The ROD system is placed between the first and the second level trigger. The data produced in the detector are gathered and digitized in the front-end electronics and transmitted to the RODs through high-speed optical links. At the first level trigger rate the ROD system has to compute in real time information from 9856 front-end channels in less than $10 \mu \mathrm{s}$. Finally, the processed data are transmitted through optical links to the Read-Out System (ROS) located in the second level trigger.
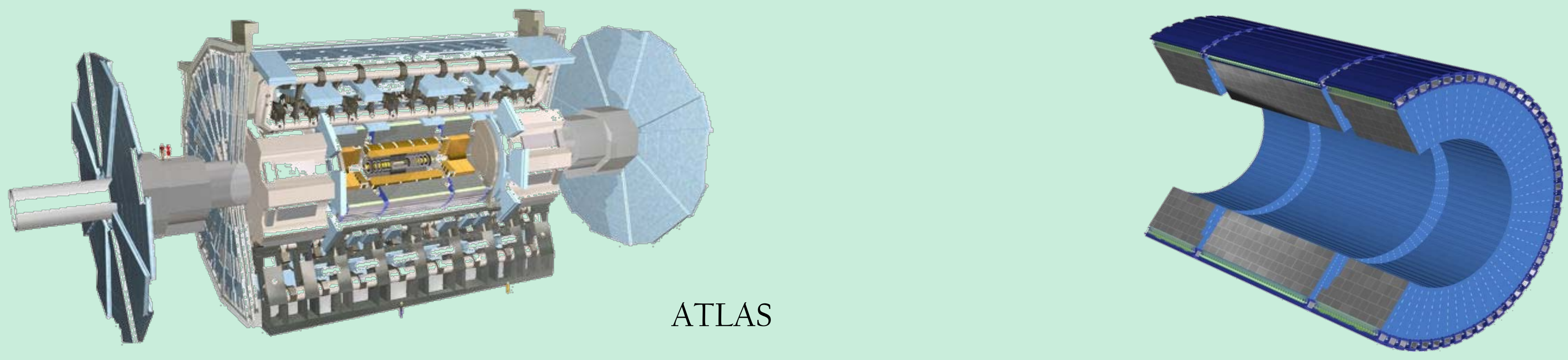

Tile Calorimeter

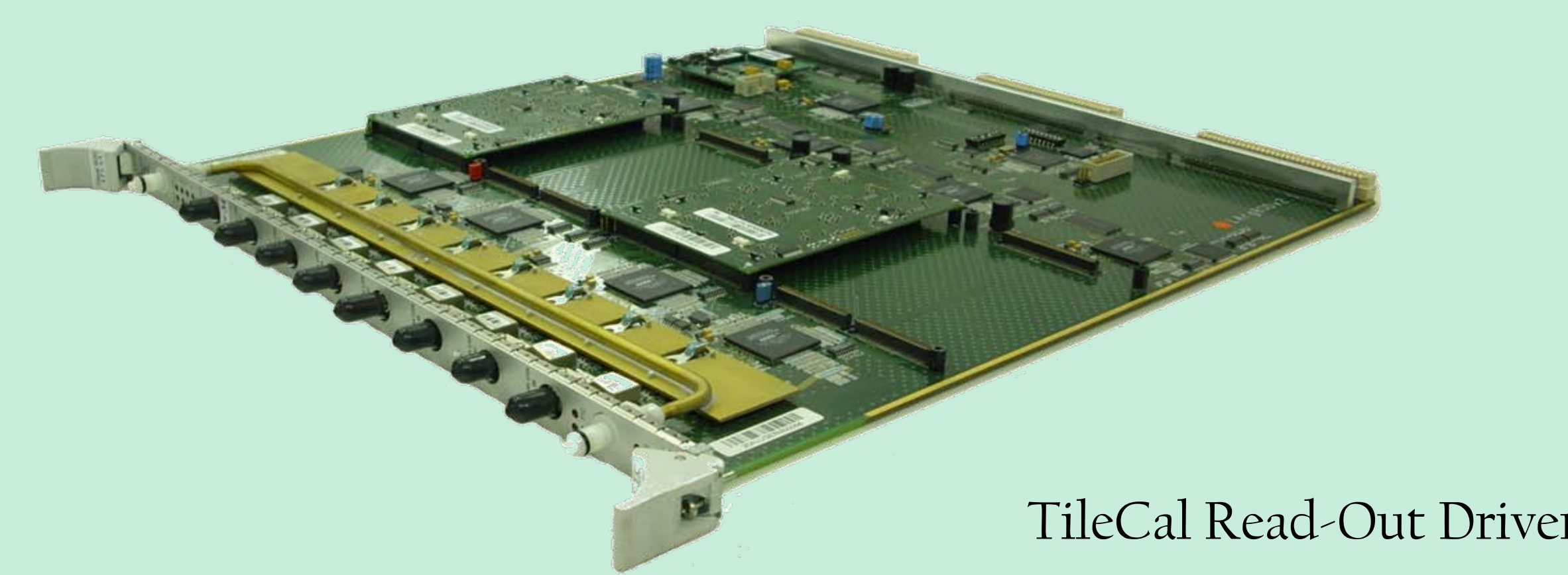

\section{Structure of the DSP code}

The main components of the TileCal ROD are the Digital Signal Processors (DSPs) placed on the Processing Unit (PU) daughterboards. The core of the DSP code acts as an operating system. It controls the configuration and manages the reception and transmission of the data. The reconstruction algorithms are performed if the front-end data is synchronized with the Timing, Trigger and Control (TTC) information.
DSP code block diagram

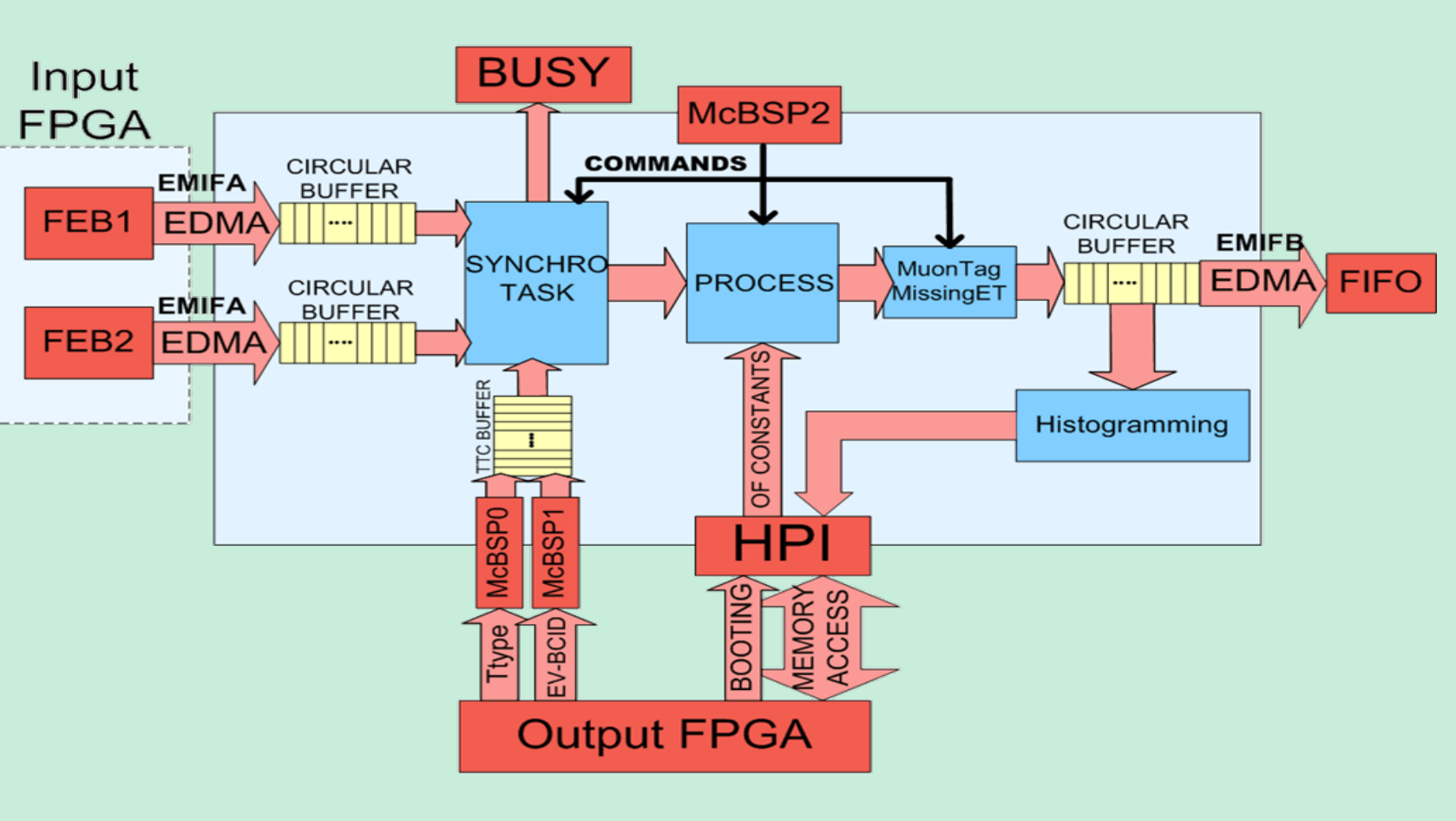

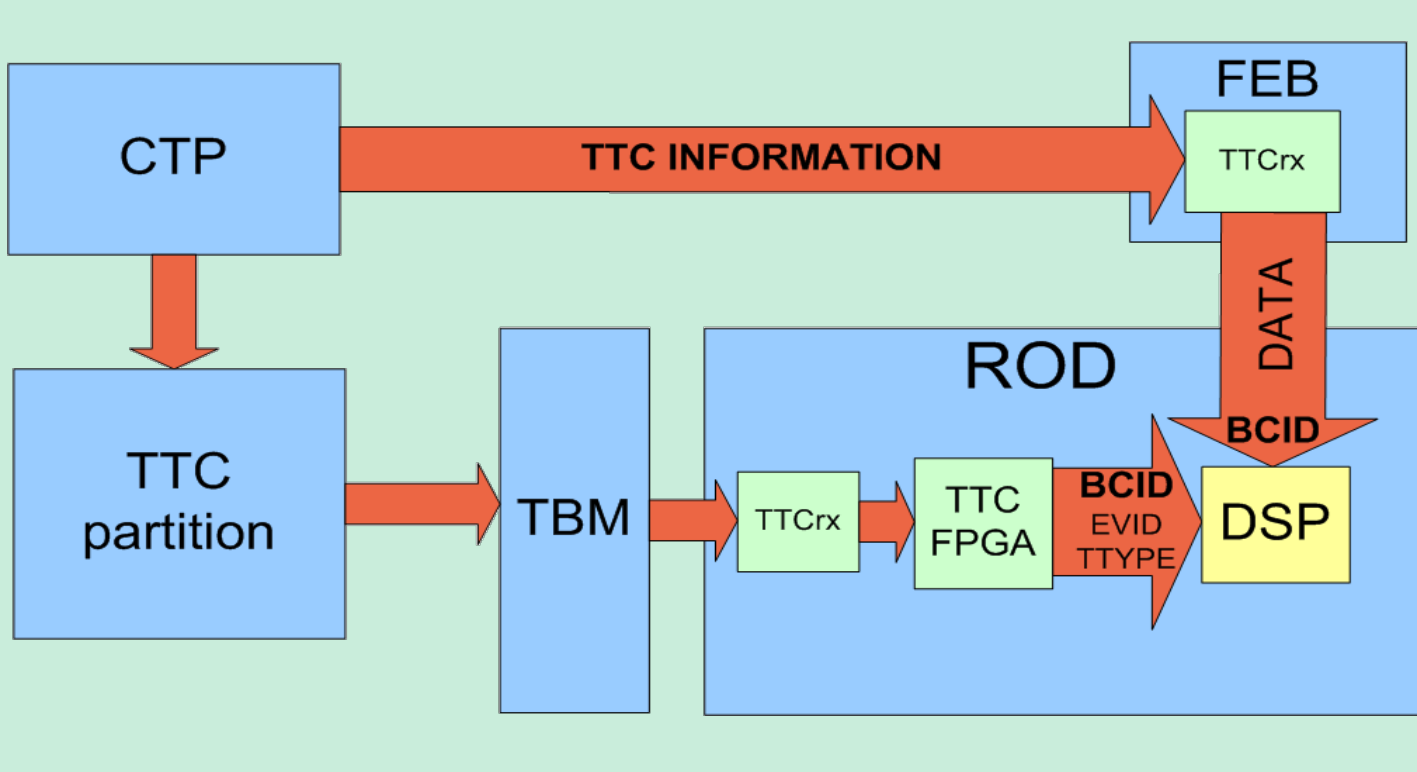

Distribution of TTC information
The DSP histogramming task monitors the OF reconstruction and the raw data at the first level trigger rate. It is the last opportunity to monitor raw data before it is replaced by OF reconstruction. It allows to online identify configuration problems and to detect digital errors.

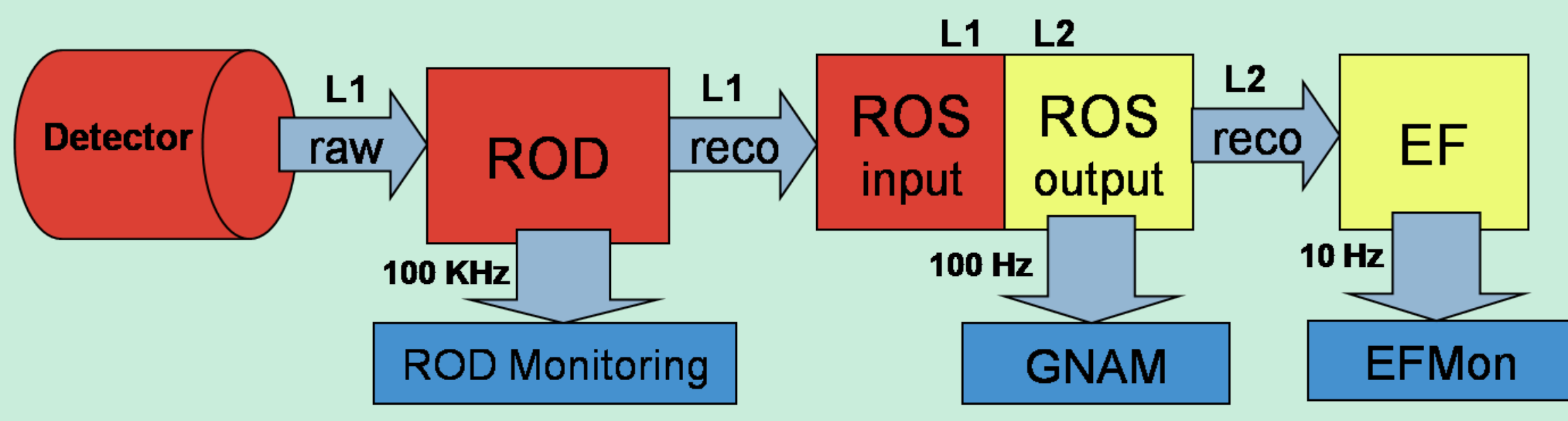

TileCal monitoring levels

The reconstruction algorithms implemented on the DSP are the Optimal Filtering (OF), Muon Tagging (Mtag) and Total Transverse Energy $\left(\mathrm{E}_{\mathrm{T}}\right)$ calculation. The OF algorithm reconstructs the deposited energy $(A)$, the phase $(\tau)$ of the signal and the Quality Factor(QF) for every calorimeter channel. This reconstructed energy is used by the MTag algorithm to tag low transverse momentum muons that may escape the ATLAS muon spectrometer Level 1 trigger whereas the $\mathrm{E}_{\mathrm{T}}$ algorithm computes the total transverse energy and the projection on $\mathrm{X}$ and $\mathrm{Y}$ axis for the entire module that will be used by the Level 2 trigger system.

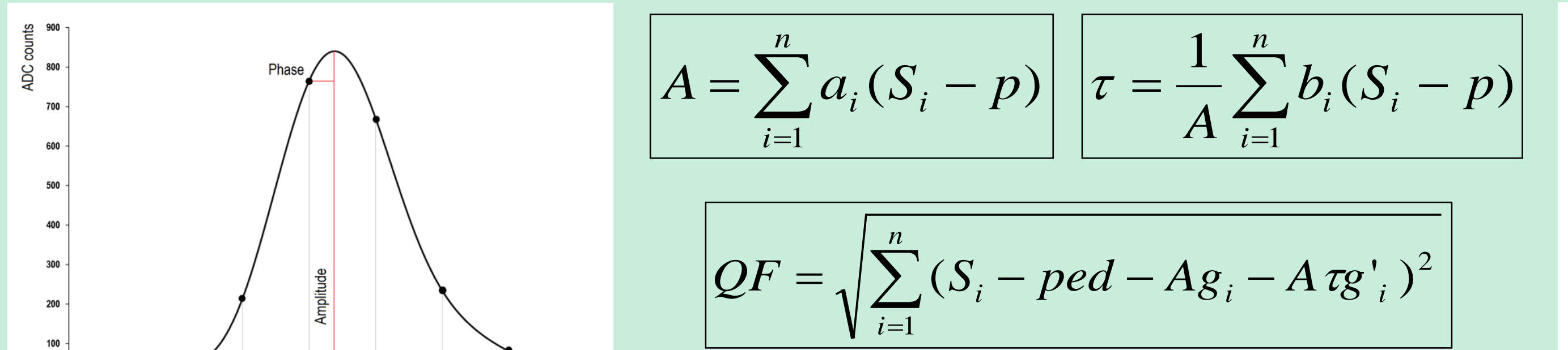

Time shape of the signal

OF definition of the energy, phase and quality factor.

The weights used in the OF algorithm are obtained from the pulse shape and noise autocorrelation matrix. The phase of the pulses during LHC operation is fixed because the digitization is synchronous with the TTC clock. Therefore, the weights are computed for the expected phase of every channel.

The performance of the OF algorithm is sensitive to phase variations. The energy reconstruction presents a parabolic deviation proportional to the phase for small phases. This deviation can be corrected offline.

\section{Signal Reconstruction Validation}

The Charge Injection (CIS) is one of the calibration systems of TileCal. It injects a configurable charge in the input of the shaper in order to calibrate the response of the readout electronics from the digitization. In addition, the injected pulse has programmable and fixed phase which allows to use the OF method. In this case the corresponding weights are retrieved for each channel from a database. The DSP reconstruction is compared with offline OF with unlimited precision. The result obtained provides a relative difference between DSP and offline below $0.4 \%$ for the energy and the absolute difference for the phase is lower than $0,02 \mathrm{~ns}$.

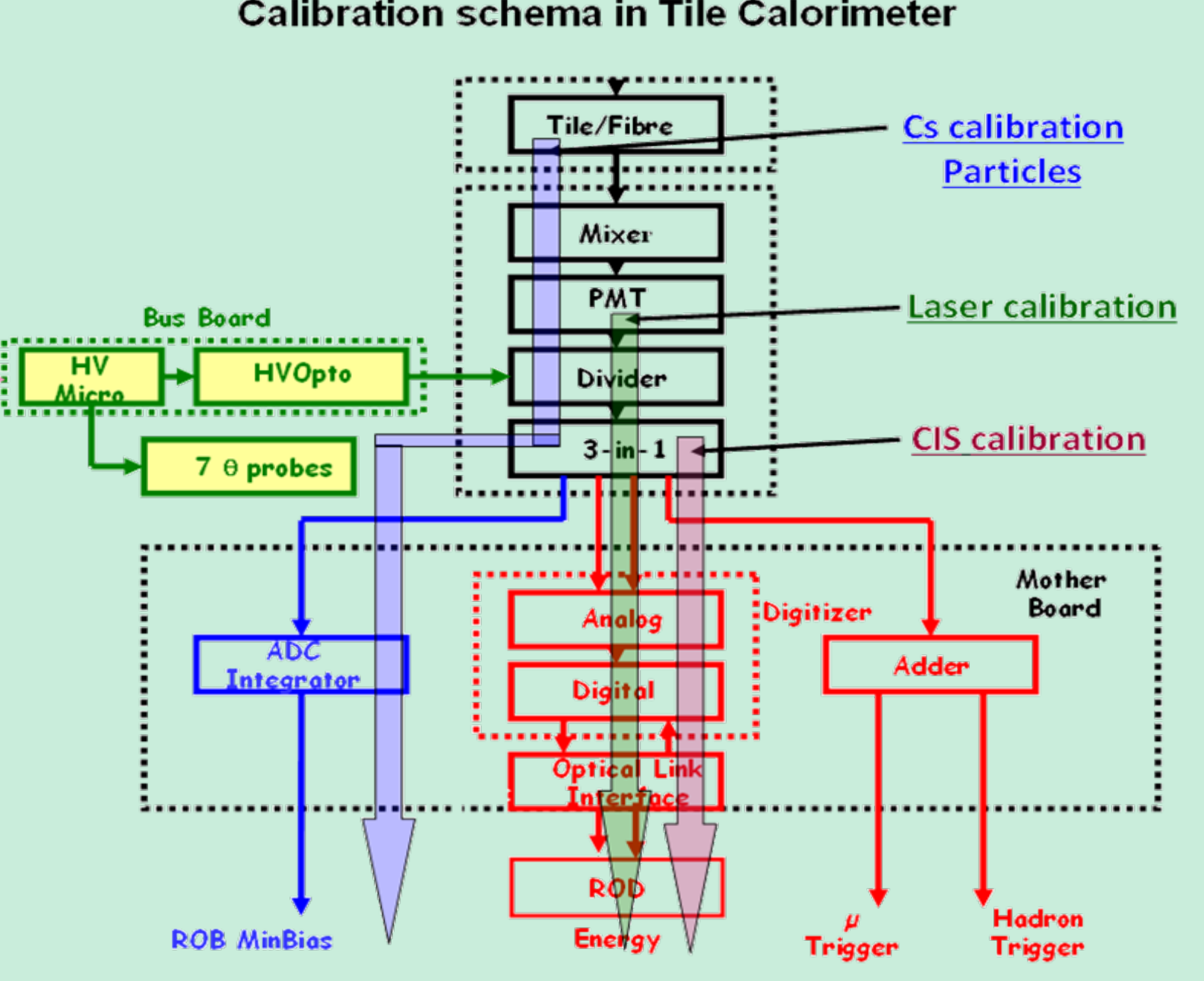

The Tile calibration relies on four dedicated systems: Cs radioactive gamma source, LASER calibration system, calibrations of digital gains and linearities with charge injection system (CIS) integrated on the drawer FE, and monitoring of beam conditions and Tile optics with the so called integrator system

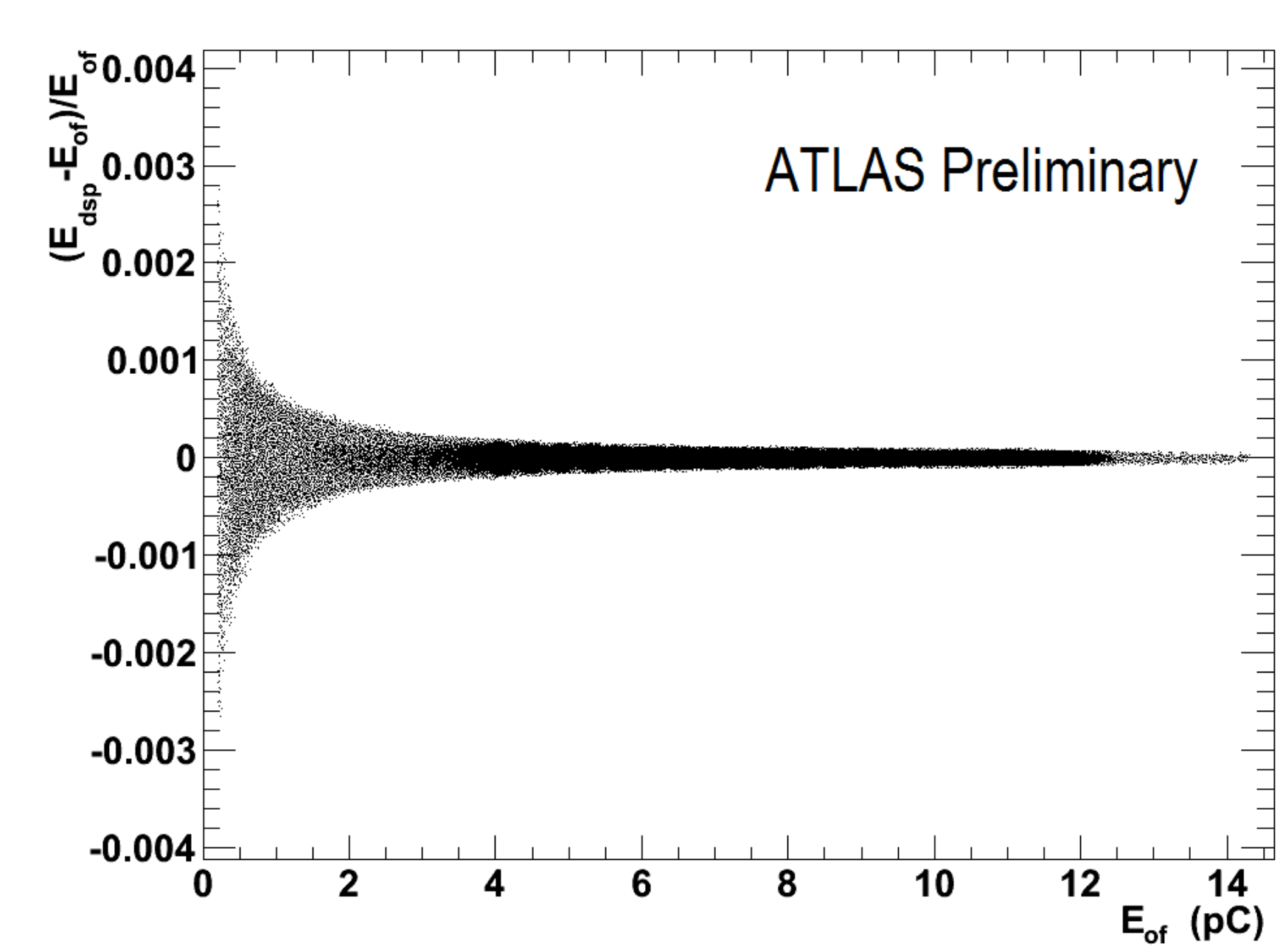

Relative difference between energy reconstructed with OF in the DSP and offline for High Gain as a function of the energy reconstructed offline.

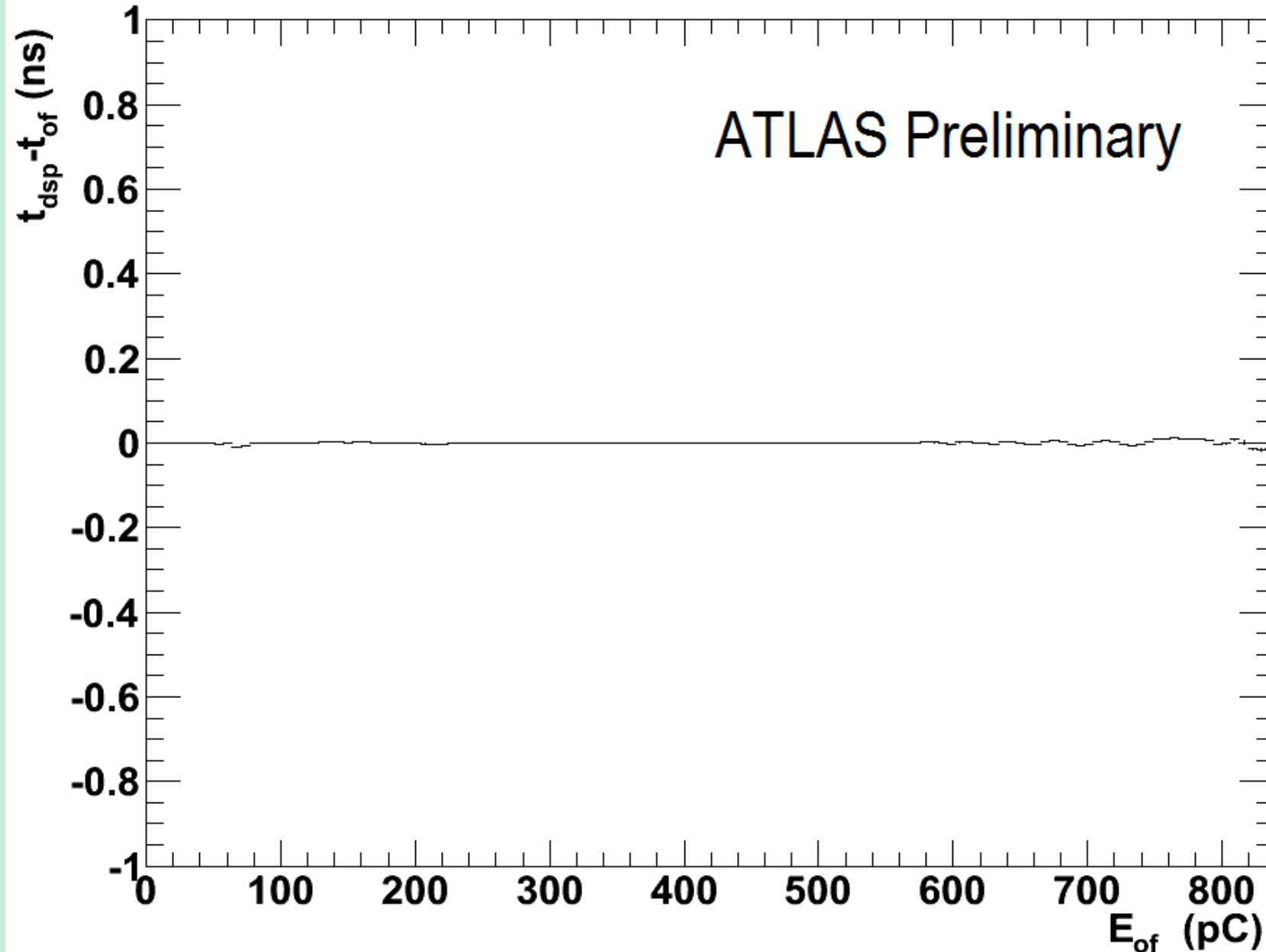

Average of the absolute difference between the phase reconstructed with OF in the DSP and offline for Low Gain as a function of the energy reconstructed offline. 